\title{
The Research of SAR Imaging Based on Co-Prime Arrays Sampling
}

\author{
Bing Han ${ }^{1,}$, Pengbo Wang ${ }^{1,2, ~ b,{ }^{*}}$, Yue Fang ${ }^{1, ~ c}$, Jincheng $\mathrm{Li}^{1, \mathrm{~d}}$, Zhirong Men ${ }^{1, \mathrm{e}}$ \\ ${ }^{1}$ School of Electronic and Information Engineering, Beihang University, Beijing, China \\ ${ }^{2}$ Collaborative Innovation Center of Geospatial Technology, Wuhan, China \\ a hanbing2015@buaa.edu.cn, b wangpb7966@buaa.edu.cn, c fangyue@buaa.edu.cn, \\ d lijincheng@buaa.edu.cn, e menzhirong@buaa.edu.cn
}

\begin{abstract}
Keywords: synthetic aperture radar (SAR), imaging algorithm, co-prime arrays, sparse sampling,
\end{abstract} compressive sensing (CS)

\begin{abstract}
High resolution wide Swath (HRWS) is one of the major development directions of synthetic aperture radar (SAR). However, the high resolution in azimuth direction and the wide range swath impose contradicting requirements on system design, and the conventional SAR systems cannot meet these rising demands. The compressive sensing (CS) theory put forward in recent years provides a way to resolve the contradiction. In the CS framework, the signal can be reconstructed with a sampling rate below the Nyquist frequency, which means that signal can be acquired and processed at a lower rate. This paper provides a sparse sampling method based on the co-prime arrays. The sparse co-prime array is utilized to reduce the echo signal sampling requirement, which can provide more signal sampling space. As a result, high resolution and wide swath can be realized simultaneously. Simulations are performed to validate the presented algorithm.
\end{abstract}

\section{Introduction}

Synthetic aperture radar (SAR) is a well-established remote sensing tool, capable of acquiring images of Earth's surface independent of weather conditions and sunlight illumination[1]. However, high geometric resolution in the azimuth direction and wide swath coverage impose contradicting requirements on system design, and the conventional SAR systems cannot meet the two requirements simultaneously. The method of CS is proposed to solve this contradiction.

In CS framework, the sampling rate required to reconstruct the original signal does not depend on the bandwidth of the signal, but on the structure and content of the information of the signal. The CS theory can be applied to avoid high-speed sampling, which will significantly reduce the cost of data storage and transmission. Using the CS theory, the signal is sampled sparsely. Generally, the reconstructed signal is not the original signal itself but the sparse representation of the original signal in the transform domain, and the sparse representation in this field contains all or enough required information for the subsequent processing. Plenty of research has been made to apply CS theory on radar imaging[2-8].

Ahmed Khwaja and Xiaoping Zhang present compressed CS imaging of SAR moving targets when there is a basis mismatch in his paper[2]. Jungang Yang and his partners propose a segmented reconstruction strategy for the CS SAR imaging scheme[3]. Junfei Chang put forward a two-dimensional SAR imaging algorithm based on CS[5] and high-resolution SAR imaging can be achieved with the algorithm. Jianping Xu researched some SAR image compression methods based on CS in his PhD thesis[8]. In the field of CS application in SAR, different sampling methods have a significant effect on SAR imaging.

Based on the CS theory, a co-prime arrays sparse sampling method is proposed in this paper. This paper proposes a working mode of SAR system and introduces the co-prime sampling theory into the SAR field. The non-uniform sparse sampling of co-prime arrays is used to reduce the requirement on sampling rate of the echo signal, which can effectively alleviate the constraint relationship between spatial resolution and the width of mapping bands. 
The rest of the paper is organized as follows. In section 2, the CS-SAR imaging algorithm based on co-prime sparse sampling is introduced in detail. In section 3, the correctness is confirmed by the result of simulation. Section 4 concludes this paper.

\section{CS-SAR Imaging Algorithm Based on Co-Prime Sparse Sampling}

\subsection{Co-Prime Arrays Sampling of SAR Signal}

In CS, the construction of the measurement matrix is equal to the sampling process, which can be achieved by random or deterministic measurement matrix. Random measurement of sampling makes a higher demand for equipment which means it is not easy to control sampling time in reality. Deterministic measurement matrix can be used to overcome the defect. In general, the entire measurement matrix is generated by a row or a line of elements which have been generated in accordance with a certain way. In this paper, the measurement matrix constructed by co-prime sampling is a deterministic matrix.

The first step of the imaging algorithm is co-prime arrays sampling. Co-prime arrays are widely used in the field of direction of arrival (DOA). A higher number of degree of freedoms (DOFs) can be achieved by exploiting a sparse array under the co-prime array equivalence. Recently several array configurations have been proposed as attractive alternatives for sparse array construction[14].

In this section, the structure of co-prime array which is used in SAR signal sampling is introduced. The prototype co-prime array is showed in Figure 2.1.

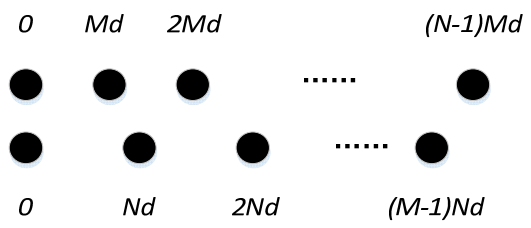

Figure 2.1 The prototype of co-prime arrays.

A co-prime array contains two uniform linear sub-arrays. One sub-arrays consists of $N$ sensors with an interval of $M \cdot d$ ( $M$ and $N$ are coprime, $d$ is the unit length); another sub-arrays contains $M$ sensors at an interval of $N d$. The structure used in this paper is illustrated in Figure 2.2, which is more common in the field of signal processing.

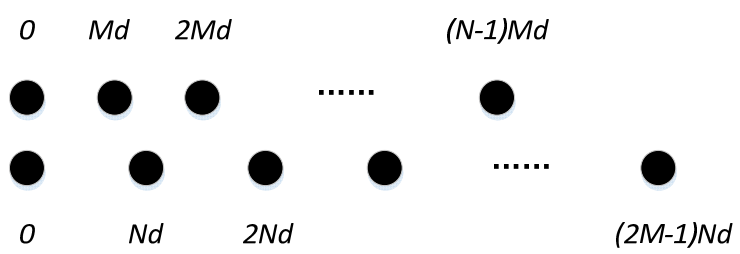

Figure 2.2 Typical coprime array

The two sub-arrays jointly own the sensor at zero position, so this array contains a total of $2 M$ $+N-1$ sensors. The freedom degree of this structure is $M N+M-1$, which is much higher than the number of sensors $2 M+N-1$. High degree of freedom means more signal information. This indicates that the sampling structure of this coherent sequence can obtain enough information of the signal by non-uniform low-speed sampling, so that the precise reconstruction of the signal is possible.

Corresponding to the sampling of the SAR echo signal, this co-prime array can be seen as the acquisition of the signal at different times, where $d$ represents the unit sampling time interval, and the position of the sensor represents a different sampling time.

SAR signal can be divided into the range and azimuth signal. Take range processing as an example, the measurement matrix is constructed as follows.

Assume that the signal echo in range direction is a discrete sequence with $N$ samples length, which is expressed as.

$$
\mathrm{E}=\left[\begin{array}{llllllllll}
a_{0} & a_{1} & a_{2} & a_{3} & a_{4} & a_{5} & a_{6} & a_{7} & a_{8} & \cdots
\end{array}\right]_{1 \times N}
$$

The sampling array is constructed by combining $a_{3}$ and $a_{5}$ as co-prime integers. So the measurement sampling array $\mathrm{Y}$ can be expressed as 


$$
\mathrm{Y}=\left[\begin{array}{llllllllll}
1 & 0 & 0 & 1 & 0 & 1 & 1 & 0 & 0 & \ldots
\end{array}\right]_{1 \times N}
$$

15 is the least common multiple of 3 and 5 .It is clear that the cycle of $\mathrm{Y}$ is 15 , and 15 is also the cycle of sparse sampling.

The measurement matrix $\phi$ is obtained by the transformation of sampling array.

$$
\phi=\left[\begin{array}{cccccccccc}
1 & 0 & 0 & 0 & 0 & 0 & 0 & 0 & 0 & \ldots \\
0 & 0 & 0 & 1 & 0 & 0 & 0 & 0 & 0 & \ldots \\
0 & 0 & 0 & 0 & 0 & 1 & 0 & 0 & 0 & \ldots \\
0 & 0 & 0 & 0 & 0 & 0 & 1 & 0 & 0 & \ldots \\
\vdots & \vdots & \vdots & \vdots & \vdots & \vdots & \vdots & \vdots & \vdots & \ddots
\end{array}\right]_{M \times N}
$$

Each line of $\phi$ has only one element 1 , whose position corresponds to the position of the element 1 in $\mathrm{Y}$, and the other elements are 0.

So the sampled SAR echo signal $\mathbf{f}$ can be expressed as

$$
\mathbf{f}=\phi \mathrm{E}^{\mathrm{T}}=\left[\begin{array}{llllllllll}
a_{0} & a_{3} & a_{5} & a_{6} & a_{9} & a_{10} & a_{12} & a_{15} & a_{18} & \ldots
\end{array}\right]_{1 \times \mathrm{M}}^{\mathrm{T}}
$$

\subsection{The Reconstruction and Pulse Compression of SAR Echo Signal}

When it comes to the SAR imaging, the construction of sparse dictionary is usually based on the linear frequency modulation characteristics of Chirp signal and matched filtering. Echo signals can be sparsely represented by constructing a convolution kernel in the time domain or constructing a reference function matrix in the frequency domain.

The SAR signal is a chirp signal with sparse characteristics after pulse compression, so the convolution kernel matrix is constructed as a sparse dictionary in time domain. The concrete construction process is as follows.

Usually an ideal chirp signal is as equation (2.5).

$$
s(t)=\operatorname{rect}(t / T) \exp \left\{j \pi k_{r} t^{2}\right\}
$$

$t$ is time. $\operatorname{rect}(t / T)$ is a rectangular function, in which $T$ means the pulse width. $k_{r}$ is linear frequency modulation. The signal is transformed to $M$ elements of the discrete sequence in digital signal processing. Suppose the echo signal reception time is $T s$ ( $T s>T$ ), it can be transformed to $N$ elements of the discrete sequence, so the sparse dictionary structure is a square which size is $N \times N$.

$$
\boldsymbol{\psi}=\left[\begin{array}{cccc}
S & 0 & \cdots & 0 \\
& & & \\
0 & S & \ddots & \vdots \\
\vdots & \ddots & \ddots & 0 \\
& & & \\
0 & \cdots & 0 & S
\end{array}\right]_{N \times N}, \quad S=s^{*}(-t)
$$

$S$ is an $M \times 1$ array, which is the time-domain convolution factor of $s(t)$.

Now, both the measurement matrix and the sparse dictionary are obtained. The last part of the algorithm is the process of reconstruction and pulse compression.

The methods of signal reconstruction based on compression perception are minimum norm model and matching pursuit class algorithm. Normally, the number of the least-norm model is extremely unstable, making it difficult to solve the problem directly. Matching tracking algorithm is widely used, the most common algorithm is the MP and OMP algorithm[8].

In this paper, we use the OMP algorithm because it is an appropriate choice in the tolerance scope. Another reason for choosing the OMP is its high computational efficiency, which is one of the advantages of SAR processing because it usually requires processing large amounts of data.

\subsection{SAR Imaging Algorithm Flow Based on Co-Prime Sampling}

In this paper, the RD algorithm is improved. The approximate process of this algorithm is showed in Figure 2.3 


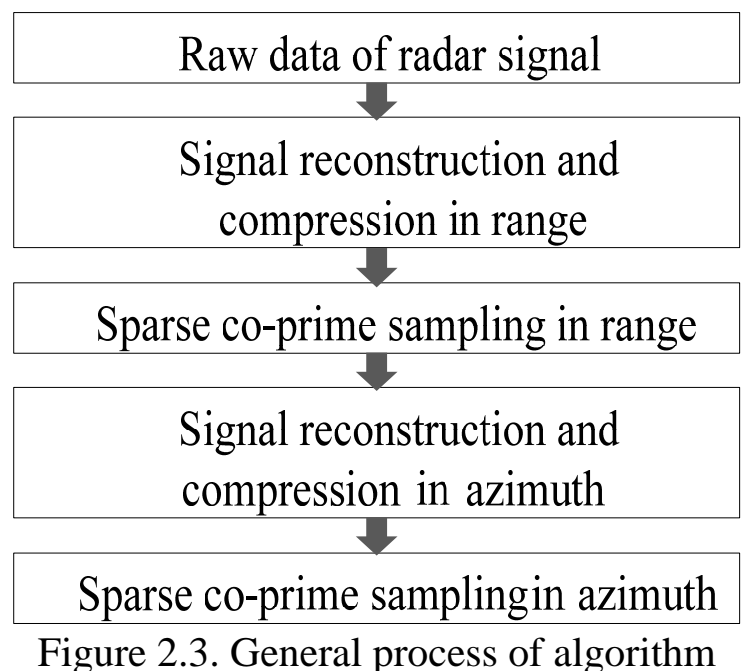

First of all, the echo signal is sampled at the sampling rate far below the Nyquist rate. Then the convolution kernel is treated as the transformation matrix in the reconstruction of the echo signal in the time domain. In fact, the pulse compression has been completed in the time domain through the transformation. Since the signal reconstructed by the OMP algorithm is in the transformed domain, the reconstructed signal has been pulse-compressed. The signal reconstruction and pulse compression are combined into one step here, which effectively improves the data processing speed. This process is respectively used both in range and azimuth direction.

\section{Simulation Data Experiments}

This section verifies the CS SAR imaging based on the co-prime sampling, which is divided into point targets and area target simulation. The modes used are on-board stripe mode.

\subsection{Simulation of Point Targets Imaging}

The parameters used are shown in Table 3.1. The co-prime numbers used are 3 and 5, and the down-sampling rate is $46.7 \%$.

Table3.1 point target simulation parameters

\begin{tabular}{|c|c|}
\hline Parameters & The parameters value \\
\hline Pulse width (s) & $10 \mathrm{e}-6$ \\
\hline Bandwidth (Hz) & $100 \mathrm{e} 6$ \\
\hline Carrier frequency (Hz) & $9.4 \mathrm{e} 9$ \\
\hline PRF(Hz) & 600 \\
\hline Flight speed (m/s) & 250 \\
\hline Flying height (m) & 20000 \\
\hline Sampling Rate (Hz) & $120 \mathrm{e} 6$ \\
\hline Target points' number in range & 5 \\
\hline Target points' number in azimuth & 5 \\
\hline Interval in range (m) & 10 \\
\hline Interval in azimuth (m) & 10 \\
\hline
\end{tabular}




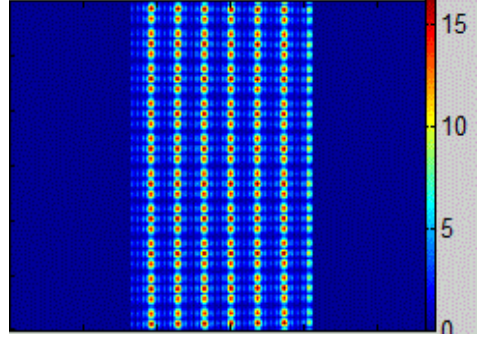

(a) Echo of point targets.

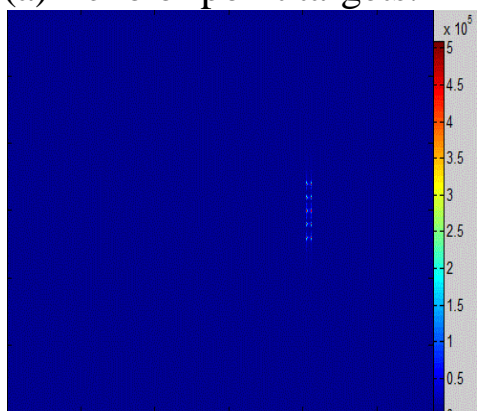

(c) 2-D compression results

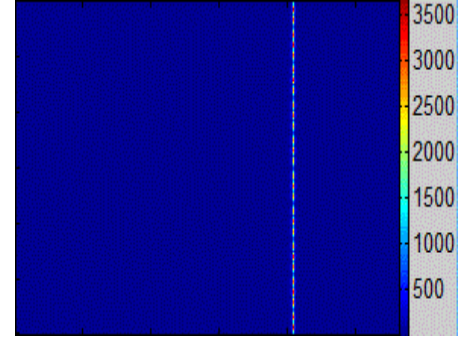

(b) Range compression results

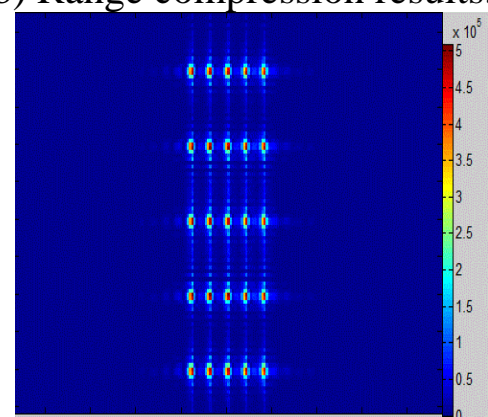

(d) magnification of (c)

Figure 3.1. The imaging results of point targets.

From the imaging results, the final image can be obtained by processing the echo data by the co-prime sampling and the compression sensing algorithm.

\subsection{The Simulation of Area Target Imaging}

The parameters used are the same as the point target simulation, with the co-prime numbers 3 and 5. In the area target simulation, the gray level of the real SAR image is used as the RCS data of the scene, the interval of range is $1.5 \mathrm{~m}$, and the azimuth lattice interval is $1 \mathrm{~m}$. The simulation result is shown in Figure 3.2.

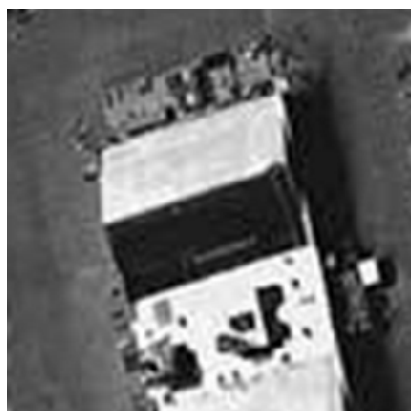

(a) Real SAR image

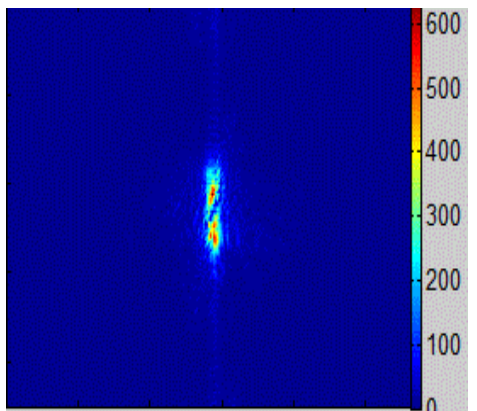

(b) Echo of area target.

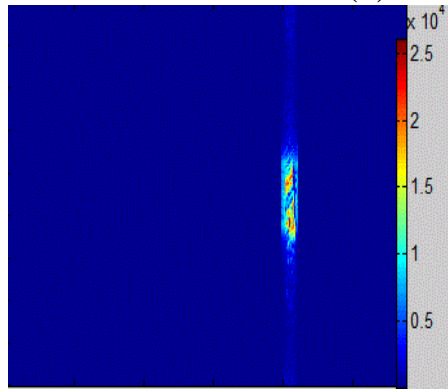

(c) Range compression result

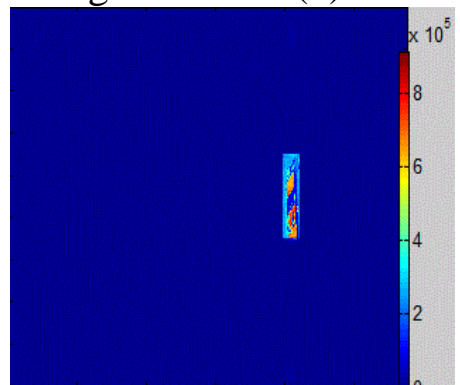

(d) 2-D compression results

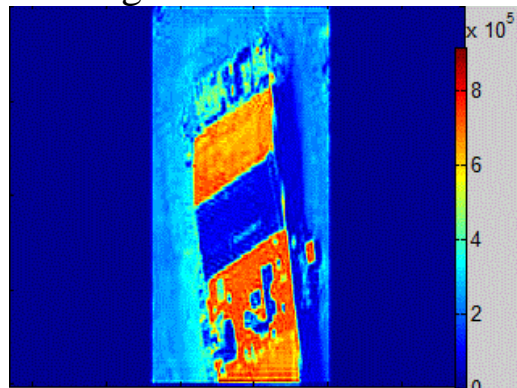

(e) Magnification of (d)

Figure 3.2. The imaging of area target

From the imaging results, it can be seen that SAR signals can be reconstructed by using the method of co-prime sparse sampling with the down-sampling rate of $46.7 \%$.

\section{Conclusion}

The high resolution and wide swath of traditional SAR mode puts forward contradictory requirements on the PRF of the radar system, and the limitation of Nyquist frequency brings great 
pressure on the sampling, storage and processing of SAR echo data. Based on the CS theory, this paper proposes a SAR imaging model based on co-prime sampling. The sampling matrix is a deterministic matrix, which solves the defect that the uncertainty matrices such as random sampling matrices in traditional compression sensing are difficult to be realized in practice. Co-prime not only breaks the limit of the Nyquist rate, but also obtains more information of the signal. The method eases the contradictions of high-resolution and wide swath. Finally, the validity of the co-prime sampling is verified by the SAR simulation of point targets and area target.

\section{Acknowledgements}

This work was supported by the National Natural Science Foundation of China (Grant No. 61628101) and the Innovation Foundation of Aerospace Science and Technology of Shanghai (Grant No, SAST2016029).

\section{References}

[1]. Curlander, J. C., McDonough, R. N. (1991) Synthetic Aperture Radar: Systems and Signal Processing. New York, NY, USA:Wiley,.

[2]. Khwaja, A., Zhang, X. P. (2013) Compressed sensing SAR moving target imaging in the presence of basis mismatch, 2013 IEEE International Symposium on Circuits and Systems (ISCAS2013), Beijing, pp. 1809-1812.

[3]. Yang, J., Thompson, J., Huang, X., Jin, T. and Zhou, Z. (2013) Segmented Reconstruction for Compressed Sensing SAR Imaging, IEEE Transactions on Geoscience and Remote Sensing, vol. 51, no. 7, pp. 4214-4225.

[4]. Jiao, L. C., Yang, S. Y., Liu, F., Hou, B. (2011) Review and Prospect of Compressed Sensing[J], Journal of Electronics, vol. 39, no. 7, pp. 1651-1662.(in Chinese)

[5]. Chang, J. F. (2012) Research on Wide Area High Resolution SAR Imaging Based on Compression Sensing[D], University of Electronic Science and Technology.(in Chinese)

[6]. Zhang, J. (2013) Research on Spaceborne SAR Imaging and Moving Target Detection Based on Compression Sensing[D], University of Electronic Science and Technology.(in Chinese)

[7]. Zhang, Q. (2009) Sparse representation based on radar signal[D], Nanjing University of Science and Technology.(in Chinese)

[8]. Xu, J. P. (2012) The Application of Compression Sensing Algorithm in Radar Imaging [D], University of Electronic Science and Technology.(in Chinese)

[9]. Cumming, I. G., Wong, F.H. (2007) Synthetic Aperture Radar Imaging - Algorithm and Implementation [M], Electronic Industry Press in Beijing.(in Chinese)

[10]. Shen, Q., Liu, W., Cui, W., Wu, S., Zhang, Y. D. and Amin, M. G. (2015)Low-Complexity Direction-of-Arrival Estimation Based on Wideband Co-Prime Arrays, IEEE/ACM Transactions on Audio, Speech, and Language Processing, vol. 23, no. 9, pp. 1445-1456.

[11]. Stojanovic, I., Çetin, M. and Karl, W. C. (2013) Compressed Sensing of Monostatic and Multistatic SAR, IEEE Geoscience and Remote Sensing Letters, vol. 10, no. 6, pp. 1444-1448.

[12]. Guo, J., Zhang, J., Yang, K., Zhang, B., Hong, W. and Wu, Y. (2015) Information Capacity and Sampling Ratios for Compressed Sensing-Based SAR Imaging”, IEEE Geoscience and Remote Sensing Letters, vol. 12, no. 4, pp. 900-904.

[13]. Guo, L., Wen, X. and Yu, J. (2013) Fast compression algorithm of SAR image based on compressed sensing, Intelligent Control and Information Processing (ICICIP), 2013 Fourth International Conference on, Beijing, pp. 144-149.

[14]. Qin, S., Zhang, Y. D. and Amin, M. G. (2015) Generalized Coprime Array Configurations for Direction-of-Arrival Estimation, IEEE Transactions on Signal Processing, vol. 63, no. 6, and pp. 1377-1390. 